\section{Versuche mit der Pasteur'schen Schutzimpfung gegen Milzbrand in Ungarn.}

\author{
Von
}

\author{
Privatdocent Dr. Aladár v. Rózsahegyi in Budapest.
}

Nach dem Behanutwerden der Impfversuche rıchtete das königl. nngar. Mınısterium für Acherban, Gewerbe und Handel an Pasten r die Auffordernng, sein Verfahren anch lner zn demonstriren. Da Pastenr von Paris nicht abkommen konnte, sandte er ejnen seiner Assistenten, Herrn Thııllıer mut den nöthigen lmpfnıaterialien nod Apparaten nach Bndapest, wo znr Beanfsıchtignng der Versnche vom Ministerinm eine ans 9 Mitgliedern bestehende Commission eingesetzt worden war ${ }^{1}$ ). Die Impfversuche wurden im September und October cr. ansgeführt nnd die Commission hat über das Ergebuıss an das Ministerium berıchtet. Bei dem allgemeinen nud gespannten lnteresse, mit welchem wissenschaftliche Kreıse dı Frage der Schntzimpfungen in allen Ländern verfolgen, dürfte es als gerechtfertigt erschenen, wenn ich es im folgenden unternehme, die Ergehnisse der in Lngarn angestellten Versuche den ansländischen Fachmännern im Wesentliclien, in freıer Bearbettnng schon jetzt mitzntheılen; der Orıginalberıcht soll sammt Beılagen vouı Mınisterınm veröffenthcht werden.

Die Bernfnng Thuillier's hatte ennen doppelten Zweck; er sollte die Sehntzkraft des Pastenr'schen Impfstoffes an Thieren, aber anch die Bereitnngsart des Impfstoffes denınstriren. Da Pasteur zn letzterem seine Eınwilligng nıcht gab, weil er Thuillier nıcht für so lange Zeıt entbehren konnte, als die Züchtnng des lmpfstoffes beansprncht und well er meinte, unsere Laboratorien seien hierzu nicht genügend eingeruchtet, rermag ich eigenthich nur über das Ergebuıss der Impfversuche Authentisches zn berichten. Obschon die Genese der Methode nnd die Zubereitung des Impfstoffes von Pasteur selbst bereits veröfentlicht worde, dürten für den anslàndischen Leser anch die mündlichen Mittheilungen nıcht uninteressant sein, welche Thnillıer hier zeitweise abgab und welche ich daher in folgenden geordnet wiedergebe.

Pasteur geht von der Beıbachtung ans, dass von Thieren, welche mit einer, Milzhradpilze enthaltenden Snbstanz (z. B. mit dem Blut an Milzbrand gefallener Sichafe) inficirt warden, der grösste Theil verendet, dıe übrıgen von der Erkranknng genesen und jetzt gegen dieselbe Mılzbrandsulsstanz nıcht melır empfänglich sind. Wenn es daher gelingen sollte, alle Thiere blos vorübergehend krank zn machen, so ware anch ıhre Immnnıtät erreicht. Zn diesem Zwecke sınd ungeschwächte Mılzbrandpilze (z. B. das erwähnte Blnt oder sporenhaltige Erde) nicht geengnet, well sle eme grössere Sterblichkeit vernrsachen, als selbst de eptzootısche Mhlzbrand. Es war daher eine so weitgehende Abschwächnng der Pllze anzustreben, dass sie nicht mehr, als eine leichte Erkrankuns an Milzbrand verursachen. Diesbezüglıch nahm Pasteur das Verhalten zu Hülfe, welches er an den Milzbrandpilzen in Knltnren beobachtete, welche bei verschiedenen $W_{\text {armegraden gehalten wurden. Die Bacllen }}$ entwickelten nnd vermehrten sich in Fleischbrühe bes $25-40^{\circ} \mathrm{C}$. am besten; ober- und unterhalb dieser Wärmegrade war die Entwickelung kümmerhch und hörte be $15^{\circ}$ nnd $45^{\circ}$ gänzlıch auf. Diese extremen Temperatnren waren nicht zn verwenden, da ja die Pilze an der Entwickelnng nucht vollkommen verhindert werden sollten: hingegen liess sıch von denjenigen, bel welchen die Entwickelung unr bümmerlich vor sıch gıng, anch eine eutsprechende Abschwäclınog der Virulenz erwarten. Gestütat auf die Thatsache, dass Vögel, insbesondere Ranbvögel gegen den Mllabrand refractär sind, nnd anf seine Versuche, welche dieses Verhalten anf die höhere, $42-43^{\circ} \mathrm{C}$. erreichende körpertemperatnr dieser Thiere znrüchfïhren, begann Pasteur einem moribunden There entnommenes nnd Mulzbrandbacillen enthaltendes Blut bei $42-43^{\circ} \mathrm{C}$. in Fleischbrühe zn zuchten und fand, dass die Bacillen jetzt keine Dauersporen mehr bilden, sondern nur zu langen homogenen Fäden auswachsen. Je länger die Pllze bei dieser Temperatur gehalten wurden,

1) Mitglieder dieser Commission sind die Universitätsprofessoren Fodór, Korányi und Plósz, pon der Thierarzueischule Director Formay, und die Professoren Azapy, Czako, Liebermanu und Than offer, und Universitātsdocent Dr. v. Rózsahegyi. nm so mehr liess ihre Virnlenz nach; während das nrsprüngliche Blnt alle mit ihm inficirten Schafe tödtete, wnrde von den, aus der Knltur am 12. Tage entnommenen Proben nnr die Hälfte der Thiere, von den am 24. Tage entnommenen gar keines mehr getödtet, aber Alle bekamen früher oder später elne geringe, etliche Tage andanernde Temperatnrerhöhnng. In den bei $42-43^{\circ}$ C. gehaltenen Knltnren blieben die Pilze 4-6 Woclien lang am Leben nnd weiter verimplbar; nach Ablanf dieser Zeit waren sie abgestorben.

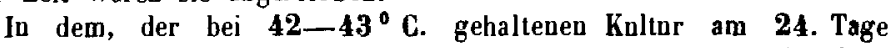
entuommenen Material war also die erwünschte abgeschwächte Virnlenz der Mizbrandpilze erreicht. Die mit diesem Stofie geimpften Thiere wurden aber von den vollkommen virnlenten ursprünglıchen Pilzen noch getödtet; hıgegen verursachte ein ans der Kultur am 12. Tage entnomnenes Material nuter diesen, mit dem 24 tägigen Stofie bereits geimpften Thieren keine Todesfalle mehr, sondern wieder nnr eine leichte fieberlıafte Erkranknng, nach deren Ablanf die Thiere nnn anch gegen die vollkommen virulenten Pilze nuempranglıch waren.

Hierın war also das Schema des Verlahrens gefnuden: Impfung mit den, durch eine 24 Tage lang bel $42-43^{\circ} \mathrm{C}$. fortgesetzte Züchtnng vollkomıen abgeschwächten Milabrandpilzen; nach Ablanf des Folgeleidens (was nach 12 Tagen stets mit Sicherheit angenommen werden kann) Impfung mit einer bei.derselben Temperatur, aber unr 12 Tage hindurch fortgesetzten, daher nur zor Hälfte abgeschwächten Knltur. Nach weiteren 12 Tagen ist anch das nach der zweiten Jmpfnng eingetretene Fieber bei allen Thieren abgelanfen, die nnn definitiv geschützt sind.

Bevor aber diese Methode in die Praxis übertragen werden konnte, waren noch zwei grosse Schwierigkeiten, die Darstellnng des Impfstoffes im Grossen nud seine Conservirnng zn bewältigen. Die bei $42-43^{\circ} \mathrm{C}$. gehaltenen Knltnren bleiben 4-6 Wochen lang am Leben; entninimt man während dieses Zeitranmes in einem beliebigen Momente eınen Tropfen, überträgt shn in reıne Fleıschbrühe nnd erhält diese constant anf $35^{\circ} \mathrm{C}$., so werden sich die Pilze hier binnen knrzem vermehrt baben, nnd dlese secnndäre Kultur wird ganz dieselbe Virnlenz besitzen, welche die primäre znr Zeit der Entualıme des übertragenen Troplens besass, - es wird also die secundäre Kultur des 12. Tages von nngeimpften Thıeren die Hälfte, die des 24. Tages aber gar keines tödten, nnd, in der nmgekehrten Rehhenfolge angewandt, werden anch diese secundären Kulturen vollkommenen Impfsclintz bieten. Nicht minder wichtıg ist es, dass die secundären Kulturen beı $35^{\circ} \mathrm{C}$. nach 48 Stunden Danersporen zn bilden beginnen und nach einigen Tagen ganz in diesen Danerznstand vibergegangen sind, dass endlich anch in diesen Sporen jener gewisse Grad der Virulenz erhalten bleibt, so dass der Impfstoff im Sporenzustande in zugeschmolzenen Röhrchen beliebige Zelt lang conservirt nnd anf jede Entferunng versendet werden kann, was bei den Fadenkulturen nıcht der Fall ist, well die Pilzfaden bei Luftabschlnss absterben, und weil der Versandt in nıcht zngeschmolzenen Röhrchen schon wegren der möglichen Verunreinignngen - nnansführbar ist. Diese Sporen können in relner Fleischbrüle wann nnd wo immer weitergezüchtet werden nnd ergeben ans geringen Mengen jede beliebige Masse eines Impfst offes von derselben Kraft.

Nachdenı die zur Schutzınıfung tanglichen Virnlenzgrade einmal errelcht nnd im Sporenzustande gewissermaassen fixirt sind, werilen nnn dıe Sclıntzimpfnngen mit späteren, xten Knltnrgenerationen des Originalstoffs ansgefülırt, wobei Pasteur, der practischen Anwendnng entsprechend, die Generationen der 24 tägigen Knltur den ersten lmpfstoff (premier vaccin), diejenıgen der 12 tägigen Kultur den zweiten Implstoff (second vaccin) nennt.

Soviel lässt sich aus den Aenssernngen von Thnillier üher die Bereitnng der lmpfstoffe entnehmen. lch gehe nun anf die Schildernng der Impfiversuche über, welche von $\mathbf{l h m}$ hier in Budapest und in der Provinz anf der Puszta Öntés bei Kapnvár (Comitat Sopron) ausgeführt wnrden.

1. In Bndapest war die Thierarzueischule der Schauplatz der Versnche. Vom Ministerium wurden Thnillier 10 Rıder 6 alte, 4 Kälber) nnd 60 Stück Schafe (30 Merinos, 30 walachischer Rasse) zur Verfügnng gestellt. Von diesen frisch eingeschafflen Thieren sollte dıe Hälfte geimpft werden, die andere nicht, und zur Controle Alle mit Milzbrand inficirt werden. Die Versnchsthiere worden von den übrigen Thieren der Anstalt, selbstredend, strenge isolirt, erhielten anch nach Arten eigene Ränmlichkeiten, wo die Rinder in einzelnen Ställen, die Schafe im selben Ranme — geimpfte nod ungeimpfte vermengt - gehaiten worden.

Während der ganzen Versnchsdaner erhielten die Thiere dasselbe Heu als Futter, welches für frei von Anthraxkeimen gehalten werden dürfte, da sich nnter den zahlreichen übrigen, mit demselben Hen gefütterten Thieren der Anstalt keine Erkrankng an Milzbrand ereignete. 2 Stück an stomatitis, wahrscheinlich in Folge von Distomnm, nud Stron- 
gylus. Zum Zweche dieser Versuche konnte man sich aher mit denı Gesundleitszustande der There zufrieden geten.

Die ron Thuillıer aus Paris in neu geschmolzenen Röhrchen mitgebrachiten Impistoffe bestanden aus sporenhaltugen Flüssigk eiten; 4 Schafe erhielten diese Stoffe unverändert ıujıcirt, die übrıgen uud die Rınder bekamen Kulturen, welche Thuillier ans jenen Sporen hier in der Thierarzneischule in sterilisirter - in zilgeschmolzenen Ballons gleichfalls aus Paris mitgebraclıter - Fleischlbrüle bei $35^{\circ} \mathrm{C}$. gezüchtet hatte und in welchen zur Zeit der Anwendung homogene und sporenbildende Fäden enthalten waren.

Die erste Schutzımpfung mit dem gänzlich abgeschwäclten Stoffe (premier vaccin) wurde an 23. Septemher an der llälfte der Versuchsthiere vorgenommen, 30 Schafe (je 15 Merinos und walachische) erlielten an der schwach behaarten inueren Schenkelfiache je $\left.1 / 6 \mathrm{Ccm}^{3}\right)$, 5 Riucler (1 Oclıs, 1 huh, 1 Fürse und 2 Kälber) in der Schnltergegend je 1/3 Ccn mit der Pravaz.'schen Spritze unter die Haut injıcirt. Nach dieser Impfung wurden weder an den Rindern, noch an den Schafen Krankheitssympitome wahrgenommen; die letzteren zeigten an den ersten zwei Tagen nicht einmal Temperaturerlöliungen. Die Thermoinetrie wurde dann, auf Thuillier's Wunsch, aufgegeben und bei späteren Impfungen überhaupt nicht mehr ausgeführt. An 8. Tage (1. October) ist ein Schaf plïtzlich verendet; die nach 7 Stunden vorgenonmene Obduction erobab Bronchialkatarrh, katarrhalısche Pneumonie, im Dünndarme Taenia expansa und Katarrh. Aus der Schenkelvene entnommene Blutund Milzpulpa wurden je einem Kanınchen in eine Haltwunde am Rücken mit negatıvem Resultat beigebracht. Thullıer setzte mit diesem Blut auch Kulturen an, erhielt aber keine Anthraxpilze.

Nach 12 Tagen (5. Octoher) bekamen die am Leben gelliebenen Thiere [29 Schafe $^{2}$ ) 2 Kälher, 3 alte Rinder] den zur Hälfte abgeschwächten Stoff (second raccin) in derselben Menge uud Gegend, aber auf der anderen Seite injicirt. Auch nach dieser Impfung traten bei den Thier en keine merklichen Erkrankungen auf, mit Ausnahme ennes Schafes, welches am 3. Tage (8. Octoher) einging. Die Obduction wurde nach 7 Stunden vorgenoinmen und ergab chronischen Magen- und Darmkatarrh, Tympanitss, partielle Schwellung aud Erweichtug der Mllz und $\mathrm{m}$ Blute Bacterien, welche den $1 \mathrm{~m}$ Impfstoff' enthalteneu ähnlıch wareu.

Da sich aus dem Sectionsbefunde nicht nut Sicherheit entnehmen liess, ob das Thier micht vielletelt doch einem Impfinilzbrande erlegen war, wurden weitere Untersuchungen angestellt. Die in Blute enthalteuen Bacillen entwickelten sıch in Hausenblaselösung ganz, wie der Bacillus anthracıs, und diese Kulturen haben zwei Kauinchen, nach subcutaner Injection, an Mhlzbraud getödtet. Das Schafblıt wurde noch zWei Kanıchen in eine Ilautwunde am Rücken gelmpft. Eines dieser Thiere wurde am 3. Tage (10. Octoher) vereudet gefunden, zegte Hyperämı der Luftwege durch Lungenodem und Im Blute Bakiterien, welche der Form und dem, in Hauseublase-Kulturen beobachteten, Entwickelungsgange nach sich als Anthiraxbacillen herausstellten. Das andere Kaunchen bheb am Leben und wies beine hrankheitserscheinuugen auf. Endlich wurden 2 Schafe mit deen schı̈äclısten Inpfstoff (premier vacciu) verselıen, und ndch 9 Tagen erhelt eiues das - bis dahin in einer sterilisirten Röhre beı Luftz.itritt gehaltene - Bhit des fraglichen Schafes, das andere ungeschwächte Mllzbrandplize unter dıe Haut gespritzt; letzteres verendete am 3. Tage an Milzbrand, das andere blieb an Leben. Alle diese Untersuchungen vermochten die Todesursache des obıgen Schafes nıcht defintiv zı entscheiden, obschion sie den auf Impfmilzbrand erhobenen Verdacht erhöhten.

Inzwischen waren auch nach der zweiten Scliutzimpfung 12 Tage abgelaufen, die Thiere waren endgältig geimpft, und es konnte die Controlinfection vorgenommen werden, welche darin bestand, dass alle geımpften, dann eine gleıche Zahl ungeimpfter Thıere eine Kultur von ungeschwächten Milzbrandpilzen unter die Haut iujicirt erhuelt. Thuıllier hatte Anthraxsporen mit sich gebracht, welche nahezu seıt 5 Jahren Im Laboratorium von Pasteur aufbewahrt waren; aus diesen bereitetet er lıer in Budapest eine Fadenkultur in Fleischbrühe und injicirte von dieser: 25 geimpften und 25 ungempften Schafen je $1 / 6 \mathrm{Ccm}$, ferner 5 geimpften und 5 ungeimpften Rundern (je zwei Kälbern und drei alten Rindern) je $1 / 2 \mathrm{Ccm}$ an der üblichen Stelle unter die Haut.

Von den Schafen zeigten die geimpften 25 Thiere an den auf die Infeetiou folgenden Tagen kemerlei Krankheitssymptome; am 7. Tage (23. 0ct.) fiel ein Stück, am 19. Tage (5. Nov.) ein zweites; die 0bduction ergab beim ersteren hochgradige Anämie und Distomum hepatıcum, bei letzterem durch Strongylus filaria bedingte Veränderungen der lungen; der Milzbrand war mit keinem Symptome vertreten.

Die ungeimpften Schafe hatten sclon nach 36 Stundeu die ersten Todesfälle und fielen in der folgenden Ordnung:

1) 4 Schafe reines Sporenmaterial, die übrigen 26 Fadenkulturen.

2) Auch diesmal dieselben 4 reines Sporenmaterial, 25 Fadenkulturen.

Deutsche Medicinische Wochenschrift. 1882.

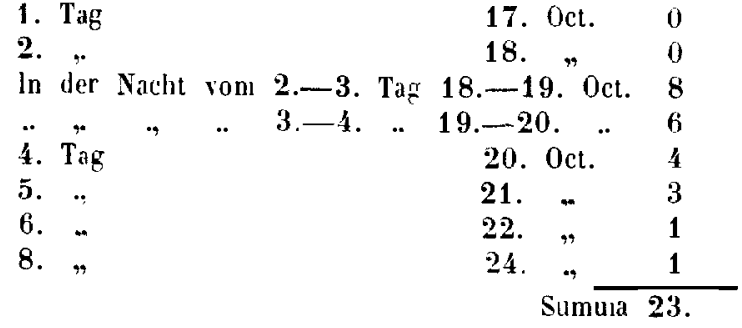

Der Leıchenbefuud entsprach bei 22 Stiick im crossen Ganzen dem Mizbrande, war jedoch in den ineisten Cadavern nıcht genügend charahteristisch ausgeprägt. In den untersichten Blutproben wurden stab- und fadenfömige (ansnalınsweise auch bewegliche) Bacterıen angetroffen. Das 23. Stück zergte gar keın Anthraxsynıtomin; das Irdrimusche, nicht coagnilirende Blut enthielt keme Bakterien: de Leber war durch Distoma hochgradıg verändert.

Von den Rındern wurde nach der Controlinfection nur an den

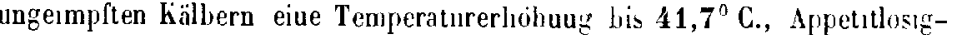
keit, trockenes Flotzmaul und rege Darmfinctiou beobichtet. Am 4. Tage waren anch diese Thuere gesund. Die grempften kälber, sov'le die reimpften und ungempften ilten Runder hessen äherhaupt keine krankilafteu Symptome beobachten.

Das Ergel)niss der Budapester Versuche lässt sich ın Foleccndeı zulsammenfassen:

Von den Schafen ist nach der ersten Schutzımpfung eln Stüek int katarrhahscher Pneumone, nach der zweiten ebenfulls ein Stuck mit Magen-Darinkatarrh gefallen; die Schutzımpfung selbst hatte also keıneu elnzigen sicheren Todesfall an Milzbrand zur Folge. Nach der Coutrolinfection verendete ein Thier mit Distomum hepat., ein zweites mit Strongylıs filaria; somit hatte unter den gempften Thıren auch die Controlinfection keınen sicheren Todesfall an Antirax rerursacht. - Die ungeimpften Schafe hatten während der Zeıt, als an den übrıgen dıe Schutzımpfungen ausgefülırt wurden, keınen einzıgen Todesfall aufzuweisen; nach der Controlinfection starben ron ibnen binnen acht Tagen $88 \%$ an Impfmilzbrand, und eln $\left(4^{\%} \%\right)$ hocligradig anamisches Stück. - Die Rinder haben die Schutzımpfung olne Verlust, ja sogar ohne Erkirankung überstanden. Nach der Controlinfection stellte sich hlos bel den ungeımpften hälbern ein kurz dauerndes Fieber eın, die geımpften Kälber, sowie die beiden alten Rinder haben auf die infection nicht einmal reagort.

2. Iclı gehe aun auf die hapuvárer Versuche iiber, welche an dres Thiergruppen ausgeführt wurden.

a. Der erste Versuch war ganz so, wie der Budapester angeordnet. Vion 100 Stick ausgenusterten Schafen wurden jo der zweımaligen Schutzimpfung uuterzogen, die anderen 50 ilcht; den Schluss bildete die Infectıon sammthcher Thıere mit tarkem Mllzbrandnıterial. Unter diesen Thieren befanden sich melirere cachectısche ludivduen: die Cachexie war ron der, in dieser Gegend grassirenden Pericarditıs ind Peritonitis bedingt. Während der Versuche wurden die Thıere in stalle gehalten und mit Hell, Stroh und Rüben gefuttert.

Die erste Schutzımpfung (mit premer vaccin) fand an 28. September statt; sie hatte weder allgemenne noch auf die lupfstelle beschränkte Erkrankungen zur Folge. Der stärkere Stoff (second valccin) wurde am 10. October verimpft, worauf sıch - ausschliessheh unter den geimpften Thieren -- die folgenden Verluste emstellten. Es fieleu:

am 1. Tage 10. October . . 0

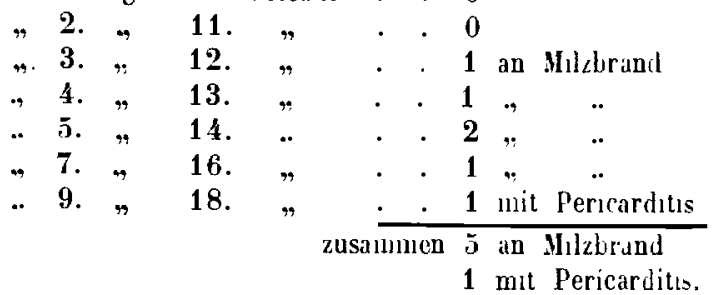

Die Controlinfection wurde an den am Leben gebhebenen 44 geimpften und den 50 ungeimpten Thieren am 22. Getoler mit folgendem Resultate ausgeführt: Von den $\mathbf{4 4}$ geimpften Thieren erkrankten an Milzbrind 3 St., wovon am 4. Tage (25. Uct.) entes verendete. Die ungempften 50 Stück fielen in der folgenden Orihung, und zeigten bei der Obduction - mit Ausnahme einger cachectıscher lndividuen ausgesprochene Nilzbrandsymptome:

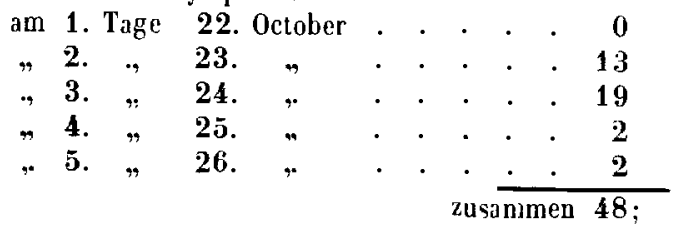

die fehlenden 2 Stück blieben am Leben. 
b. In Kapuvár wurde mit einer 489 Stück zählenden Schafherde der Versuch in der Weise angeordnet, dass etwa die Hälfte die doppelte Schutzimpfung erlitt und dann die ganze Herde nicht künslich inficirt, sondern der Einwirkung des natürlichen Milzbrandcontagiums ausgesetzt wurde, welches vor der Impfung in der Herde wöchentlich 2 bis 3 Thiere tödtete. Das Material und die Tage der Impfung sowie die Wartung der Thiere wie bei Versuch a.

Die erste Schutzimpfung wurde an 267 Thieren ausgeführt, wovon eines kurz nach der Injection an Milzbrand fiel, als Zeichen dessen, dass es bereits in inficirtem Zustande zur Impfung gekommen war. Zwei andere Thuere erlagen nach 4-5 Tagen gleichfalls an Milzbrand; von diesen Fällen lässt sichı nicht entscheiden, ob die Infection erst durclı die Impfung oder schon früher, auf natürlichem Wege erfolgte. Es werden daher alle dreı Fälle als zweifelhaft mit einem (?) zu versehen sein. Während derselben Zeit war auch von den ungejmpften 222 Thieren eines an Milzbrand gefallen.

Nach der zweiten Schutzimplung wurden an Milzbrand die folgenden Todesfälle verzeichnet:

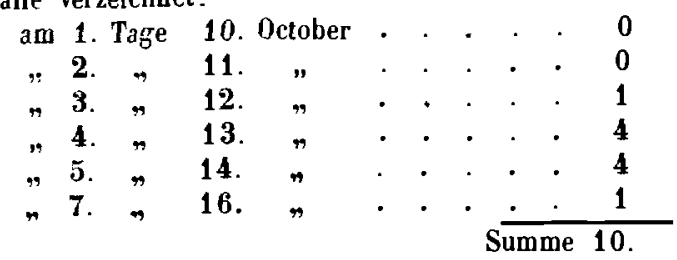

Ausserdem traten beı 12 von den überlebenden 254 Stück an der Injectionsstelle entziindliche Infiltrationen auf, welche sich auf den ganzen Schenkel verbreiteten und zur Bildung von faustgrossen Abscessen, Fisteln und Hohlgängen fülırten, in Folge dessen die Thiere an den ergriffenen Extremitäten hinkend blieben.

Die überlebenden 254 geimpften +221 ungeimpften $=\mathbf{4 7 5}$ Schafe werden seitlem auf ihrer gewohnten Weide gehalten, und soll die weitere Beobachtung zeigen, ob die geimpften Thiere vom natürlichen Milzbrandcontagium weniger zu leiden haben, als die ungeimpten.

c. Der dritte Versuch wurde in Kapuvár an $\mathrm{h}$ indern angestellt. Von 10 Stück ungarischer Rasse und 10 Stück gemischter Abkunft wurden je 7 mil Schutzimpfungen versehen, je 3 aber nicht, und zum Schluss erfolgte die Controlinfection sämmtlicher 20 Thiere mit ungeschwäclıtem Anthraxmaterial. Unter diesen Thieren waren nur zwel der ungeimpft gebliebenen abgemagert, sonst aber gesund; die übrigen waren gut genährt und alle entstanmten einer gesunden Herde. Während des Versuches wurden sie wie die Schafe gehalten; die Versuchsanordnung entsprach der lu Budapest befolgten; Impftage und Impfstoffe wie bei Versuch $a$ und $b$.

Nach der ersten Scliutzimpfung konnte an den 14 Thieren nichts Alnormes beobachtet werden; nach der zweiten war ein Stick von gemiscliter Abkunft einen Tag lang traurig und appetitlos. An diesen geimpften Thieren hatte auch die Controlinfectıon keine weiteren Folgen, als dass en Stück von gemischter Abkunft am ersten Tage traurig war und die Nalırung verweigerte; von den ungeinipften 6 Thieren - welche sich bisher ganz wohI befunden hatten - sind hingegen nach der Infection 4 Stïck scliwer erkrankt. Die Symptome bestanden in lohem Fieher, ausgebreiteter entzündlicher Infiltration der Impfstelle, profusen Schwerssen, Injection der Scheidenschleimlıaut und blutig-schleimigem Ausflusse aus der Scheide, Appetitlosigkett, trägen Darmentleerungen und Muskelzıttern. Eines dieser Thiere (eine 13 jährige Kuh von gemischter Abkunft) ist am 7. Tage an Impfmizbrand eingegangen.

Das Ergebnıss der in Kapuvár ausgeführten Versuche lässt sich in Folgendem zusammenfassen: Von den insgesammt 317 Schafen fielen nach beiden Schutzimpfungen 18 Stück $(5,68$ Proc.) an Milzbrand, eines (0,32 Proc.) mit einer anderen Krankheit, ausserdem wurden 12 Stück lahm. Von den zu Controlversuchen bereit gehaltenen 272 Thieren starb wäbrend derselben Zeit nur eınes (0,37 Proc.) an Milzbrand, an anderen Krankheiten gar keines. Nach der Controlinfection waren von $44 \mathrm{ge}-$ impften Thieren an Milzbrand 3 Stück (6,8 Proc.) erkrankt und von diesen eines (2,27 Proc.) verendet; von den 50 ungeimpften Schafen wurden durch die Infection 48 Stūck (96 Proc.) getödtet, wie die 0 bduction ergab, mit Ausnahme einiger cachectischer Individuen, an ausgesprochenem Milzbrand ${ }^{1}$ ). Beim Rindvielı verlief die erste Scliutzimpfung ohne merkliche Folgen, die zweite verursachte bei einem Thiere $(1 / 1=7,1$ Proc. $)$ eine leichte Erkrankung. Auch nach der Controlinfection wurde unter diesen geimpften Thieren nur eine leichte $\mathrm{Er}$ krankung $(7,1$ Proc.) beobachtet, hingegen waren von den 6 ungeimpften Thieren 4 Stück $(66,7$ Proc.) schwer erkrankt, und eine von ihnen (16,7 Proc.) verendete an Milzbrand.

Wir wollen nun aus diesen, auf der beiliegenden Tabelle zusammengestellten Versuchsergebnissen alle zulässigen Folgerungen ziehen. So wie der Commissionsbericlit, werde auch ich die mit derselben Methode anderwärts erzielten Resultate unberücksichtigt lassen.

Die pathologische Wissenschaft wird es als eine grosse Errungenschaft betrachten, dass wir durch diese Versuche in Stand gesetzt werden, Pilze, welche uns als ständige Begleiter einer tödtlichen Infectionskrankheit bekannt sind, durch künstliche Züchtung in ihrer Wirkung soweit abzuschwächen, dass sie den Organismus, welchem sie einverleibt wurden und in welchem sie sich vermehrt haben, blos vorübergehend krankmachen, dadurch aber auch seine Empfänglichkeit gegen Pilze von der ursprünglichen Virulenz aufheben. Hierin liegt einerseits der Beweis dessen, dass wirklich die Bacterien die krankmachenden und tödtenden Agentien sind, - andererseits auch eine Erklärung für die Wirkungsart der Schutzimpfungen, welche gegen andere Krankheiten (z. B. Menschenpocken) schon seit langem in Anwendung stehen, ohne dass wir vermocht hätten, uns thre Wirkung zu erklären.

Von grösserer Wichtigkeit sind die practischen Resultate dieser Versuche, weil hier die Gesundhetspflege und Oeconomie, also Gesundheit und Vermögen in hohem Maasse interessirt sind. Dieser Umstand möge meine Ausführlicłıkeit entschuldigen.

Pasteur stellt das unmittelbare Ergebniss der Controlinfection in den Vordergrund, und wenn auch wir so vorgehen, so lässt sich darauf hinweisen, dass in Budapest und Kapuvár nach der Controlinfection von 69 geimpften Schafen ein einziges (1,45 Proc.), von 75 ungeimpften Thieren hingegen 70 (93,3 Proc.) an Mlzbrand verendeten. Der Unterschied ist so eclatant, dass er jeden Zweifel an der unmittelbaren Ausführbarkeit allgemeiner Schutzimpfungen geradezu auszuschliessen scheint, und man geräth gewissermaassen in den Verdacht des Uebelwollens, wenn man sich an dieses Ergebniss mit Entgegnungen heranwagt.

Und doch lässt sich aus unseren Versuchen, wenn man ihren ganzen Verlauf berücksichtigt und sich nicht blos auf die Schlussscenen beschränkt, noch vieles Andere herauslesen.

Erstens ist bereits nach den Schutzimpfungen ein Theil der Thiere mit den Symptomen des Milzbrandes verendet. Wenn es aucl fraglich blieb, ob der eine Budapester Fall dem Milzbrand erlag und ob in den ersten drei Kapuvárer Fällen die Infection mit dem als sichere Todesursache erkannten Milzbrand nicht schon vor der Schutz-

1) Da nichts hestimmt angegeben wurde, wie viel nnter diesen ,einigen cachectischen Individuen zu verstehen sind, musste ich die ganze Procentzabl in die Rubrik "Milzbrand “ aufnebmen, wo ich sie aber, zum Zeichen dessen, dass sie auch ohne Milzbrandsymptome gefallene Thiere entbält, mit einem (?) versehe.

Sterblichkeit der Versucbsthiere pro 100 (berecbnet).

\begin{tabular}{|c|c|c|c|c|c|c|c|c|c|c|c|c|c|c|c|c|c|}
\hline & \multirow{4}{*}{$\begin{array}{c}\text { Versuch } \\
0 \text { Milzbrand. }\end{array}$} & \multicolumn{10}{|c|}{ Nach den Schutzimpfungen. } & \multicolumn{4}{|c|}{ nach der Controlinfection } & \multirow{2}{*}{\multicolumn{2}{|c|}{$\begin{array}{c}\text { Gesammt - Sterb- } \\
\text { lichkeit }\end{array}$}} \\
\hline & & \multicolumn{4}{|c|}{ Erste Impfung } & \multicolumn{4}{|c|}{ Zweite Impfung } & \multicolumn{2}{|c|}{ Zusammen } & \multicolumn{2}{|c|}{ geimpfte } & \multicolumn{2}{|c|}{ ungeimpfte } & & \\
\hline & & \multicolumn{2}{|c|}{ geimpfte } & \multicolumn{2}{|c|}{ ungeimpfte } & \multicolumn{2}{|c|}{ geimpfte } & \multicolumn{2}{|c|}{ ungeimpfte } & \multirow[b]{2}{*}{ geimpfte. } & \multirow[b]{2}{*}{$\begin{array}{l}\text { unge- } \\
\text { impfte. }\end{array}$} & \multirow[b]{2}{*}{$\begin{array}{c}\text { Milz- } \\
\text { brand. }\end{array}$} & \multirow[b]{2}{*}{$\begin{array}{l}\text { andere } \\
\text { Krkb. }\end{array}$} & \multirow[b]{2}{*}{$\begin{array}{l}\text { Milz- } \\
\text { brand. }\end{array}$} & \multirow[b]{2}{*}{$\begin{array}{l}\text { andere } \\
\text { Krkh. }\end{array}$} & \multirow[b]{2}{*}{ geimpfte } & \multirow[b]{2}{*}{$\begin{array}{l}\text { unge- } \\
\text { impfte. }\end{array}$} \\
\hline & & $\begin{array}{l}\text { Milz- } \\
\text { brand. }\end{array}$ & $\begin{array}{c}\text { andere } \\
\text { Krkb. }\end{array}$ & $\begin{array}{l}\text { Milz- } \\
\text { brand. }\end{array}$ & $\begin{array}{c}\text { andere } \\
\text { Krkb. }\end{array}$ & $\begin{array}{c}\text { Milz- } \\
\text { brand. }\end{array}$ & $\begin{array}{l}\text { andere } \\
\text { Krkh. }\end{array}$ & $\begin{array}{l}\text { Milz- } \\
\text { brand. }\end{array}$ & $\begin{array}{l}\text { andere } \\
\text { Krkb. }\end{array}$ & & & & & & & & \\
\hline 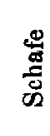 & $\begin{array}{l}\text { Budapest } \\
\text { Kapuvár (a) } \\
\text { Kapuvár (b) } \\
\text { Mittel }\end{array}$ & $\begin{array}{c}0 \\
0 \\
1,12 \\
-\end{array}$ & $\begin{array}{c}3,33 \\
0 \\
0 \\
-\end{array}$ & $\begin{array}{c}0 \\
0 \\
0,45 \\
-\end{array}$ & $\begin{array}{l}0 \\
0 \\
0 \\
-\end{array}$ & $\begin{array}{c}3,45(?) \\
10,0 \\
\left.3,79^{2}\right) \\
-\end{array}$ & $\begin{array}{c}0 \\
2,0 \\
0 \\
-\end{array}$ & $\begin{array}{l}0 \\
0 \\
0 \\
-\end{array}$ & $\begin{array}{l}0 \\
0 \\
0 \\
-\end{array}$ & $\begin{array}{r}6,78 \\
12,0 \\
4,87 \% \\
6,05\end{array}$ & $\begin{array}{c}0 \\
0 \\
\mathbf{0 , 4 5} \\
\mathbf{0 , 8 3}\end{array}$ & $\begin{array}{c}0 \\
\left.2,27^{1}\right) \\
-4\end{array}$ & $\begin{array}{r}8,0 \\
0 \\
-\end{array}$ & $\begin{array}{r}88,0 \\
96,0(?) \\
-0\end{array}$ & $\begin{array}{r}4,0 \\
0 \\
-\end{array}$ & $\begin{array}{c}14,78 \\
14,27 \\
14,53\end{array}$ & $\begin{array}{c}92,0 \\
96,0 \\
\overline{94,0}\end{array}$ \\
\hline 恋 & $\begin{array}{l}\text { Budapest } \\
\text { Kapnvar (c) } \\
\quad \text { Mittel }\end{array}$ & $\begin{array}{l}0 \\
0 \\
-\end{array}$ & $\begin{array}{r}0 \\
0 \\
-\end{array}$ & $\begin{array}{l}0 \\
0 \\
-\end{array}$ & $\begin{array}{l}0 \\
0 \\
-\end{array}$ & $\begin{array}{l}0 \\
05 \\
-\end{array}$ & $\begin{array}{l}0 \\
0 \\
-\end{array}$ & $\begin{array}{l}0 \\
0 \\
-\end{array}$ & $\begin{array}{l}0 \\
0 \\
-\end{array}$ & $\begin{array}{l}0 \\
0^{6} \\
0\end{array}$ & $\begin{array}{l}0 \\
0 \\
0\end{array}$ & $\begin{array}{l}0 \\
07 \\
0\end{array}$ & $\begin{array}{l}0 \\
0 \\
0\end{array}$ & $\begin{array}{c}04) \\
\left.16,7^{\circ}\right) \\
9,1\end{array}$ & $\begin{array}{l}0 \\
0 \\
0\end{array}$ & $\begin{array}{l}0 \\
0 \\
0\end{array}$ & $\begin{array}{c}0 \\
16,7 \\
9,1\end{array}$ \\
\hline
\end{tabular}

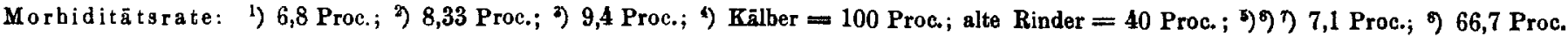


impfung erfolgt war, so fielen doch am letzteren 0rte 15 Thiere ganz sicher jenem Milzbrand zunı Opfer, welcher nur auf dıe zweite Schutzimpfung zurüekgefühırt werden kann. Es sst mojglich, dass der lier zur Verweudung gekommene lmpfstoff stärker war, als dass die, erst mit dem schwächsten Stoffe geimpften Thiere ıhn hätten ertragen küunen; auch das ist nicht ausgeschlossen, dass sich bei der Belıandlung des Inıpfstoffes oder beim Impfverfahren Unreingkeiten eingeschhichen hatten, und dass sıch zum Milzbrand auch noch ene septische lnfection gesellte. Als Entschuldigungsgrund kann alles das niclıt gelten, denn man darf nicht übersehen, dass man es hier mit Musterversuchen zu thun hat, welclıe in jeder Bezichuug mıt der grössten Vorsıcht, gewıssermaassen als theoretısche Demonstrationen ausgefülırt wurden und man soll nicht vergessen, dass eine jede Methode, jedes theoretısch festgestellte Verfahren, jede Construction durch die allgemeine Anwendung in der alltäglıchen Praxis sehr viel an Reinlıeit und Präcision einbüsst. Darum stelit heute noch zu befürchten, dass sich in der lmpfpraxis milabrandige und septische infectionen sebr leschi und darun nicht selten einstellen werden.

Auch das wird der Aufmerksamkelt nicht entgangen seln, dass nach der Schutzımpfung an anderen krankheiten - richtiger: dass mit den Leichensymptomen von anderen krankheiten (katarrh. Pneumonie, Distomum, Strongylus, Pericarditis) und nicht vou Antlirax - ausschliesslich die gempften Thiere erlagen. Es folgt lieraus, dass durch die Schutampfung der letlale Ausgang anderer latenter schwerer Leiden des körpers beschleunigt wird.

Vonı practisclien Gesichtspunkte kommt es so ziemlich auf eines heraus, ob der Verlust durch Milzbrand oder durch andere hrankheiten verursaclıt ist; darum sind die letzteren Todesfälle den von Anthrax verursachten zu subsummiren. Thut man es und addirt man auch sämmtlıche sowohl nach den Schutzimpfungen, wie nach der Controlinfection eingetretenen Todesfälle, so erhält man als Hauptergebniss, dass von den ungeimpften Thieren 94 Proc., von den geınipften 14,5 Proc. erlegen sind.

Der Unterschied bleibt auch so noch sehr bedeutend; $1 \mathrm{mmerlin}$ ist aber auch die Sterblichkeıt der geinpften Thiere (14,5 Proc.) keıne gerınge. Diese Sterblichketsrate war in zwei Versuchen so nahe übereınstimmend $(14,78$ und 14,27 Proc.), dass sie beinahe als Regel erschennt, weslaalb auch nicht anzunelımen ist, dass in grossem Maassstab ausgeführte Impfungen en günstigeres Resultat liefern werden. Dabei durf aucht vergessen werden, dass hier Durchschnittszahlen vorlıegen, während nıan es in der Praxıs mıt Einzelfällen zu thun hat; in solehen Fällen aber kanı dıe von der Schutzımpfung abhängige Sterbliclıkeit an Mlızbrand alleın - wile dıe obıgen Versuche zeigen - von 3,5-10 Proc. der geimpften There ansteigen. Zieht man alles das in Betracht, so ist die Befürchtung nıcht unbegründet, dass in der Praxis ein noch hoherer Sterblichkeitssatz sich argeben wird, als der obıge ist. Andererseıts dürfen, vonı practischen Gesichspunkt, dieser Sterbhichkett nıclit die obıgen 94 Proc. der ungeimpften Thıere, sondern nur jene Mortalıtätsraten gegenüber gestellt werden, unter welchen dıe Herden in Mılzbrandgegenden zu leıden haben, welche doch bedeutend niedriger sind.

Es stelıt hier ausser Frage, dass selbst eıne Sterblıchlieit von 14,כ Proc. noclı annelımbar wäre - da ja der durch Mizbrand verursachte jährliche Verluste in einzelnen Gegenden sogar 60 Proc. erreicht - wenn durch sie nur eın sicherer Schutz erkauft werden könnte, eln Schutz gegen das natürlıche Milzbrandcontagium.

ln eınem der hapuvárer Versuche waren von den zweımal germpften Schafen nach der Controlınfection 3 Stück an Milzbrand erkrankt, wovon eines verendete; der lnpfschutz ist also kein vollkonmen sicherer. Da jedoch diese Fälle bisher vereinzelt stehen, berechtigen sie zu kennen weiteren Folgerungen; bei der Resumırung des Ergebnisses werden sie keinesfalls der Zweckmässigkeit der Verallgemeınerung der Nethode das Wort reden. Als Bewess dessen, dass der Impfschutz in Wirklichkeit gegen das wahre Mllzbrandcontagium geboten wird, weist man uns die Wirkung der Controlinfection auf ungeimpfte Thiere vor. Der Krankheitsverlauf, der Sectionsbefund, insbesondere auch die gefundenen Bacillen, lassen keinen Zweifel darüber aufkomnien, dass zur Controlinfection Anthraxmaterial verwendet worden war; die Beobach-tungen aber, dass die Rinder nicht einmal alle davon erkrankten und ganz ausnahmsweise davon starben, - ferner, dass (besonders in Budapest) das Absterben der Schafe sich auf eınen langen Zettraum hinzog, und dass die Obduction dieser Thiere keine gut ausgeprägten Milzbrandsymptome ergab, scheinen dafür zu sprechen, dass das zur Controlinfection verwendete Material von etwas milderer Virulenz war als das natürlıche Milzbrandcontagium. Inwiefern die Schutzkraft dieser Impfungen auch gegen die in der Natur verbreiteten Milzbrandkeime Gültigkeıt besitzt, das wird sich erst nach Beendigung des nuit b. bezeichneten Kapuvárer Versuches beurtheilen lassen; es werden nämlich dort 254 Stück geimpfte und 221 stück ungeimpfte Thiere auf bekannten Mizbrand- weiden gehalten. lnzwischen liat aber mit Eintrilt des Winters der epizootische Milzbrand überhaupt beinahe ganz aufgehort, und wird erst in der warmen Jahreszeit wieder solche Dimensionen annelınen, um seine Wirkungen an diesen herden Thergruppen vergleichen zu bönnen. Bıs dahin kan es nucht für bewiesen gelten, dass diese lmpfungen auch gegen das natürliche Mlabrandcontagium soviel Schutz bieten, als gegen das zur Controlinfection angewendete aus Jahıre alten Sporen gezüchtete Material.

Die Methode bedarl also noch solcher Modificationen, dass die Schutzımpfung selbst keine Sterbhıchkeit verursache, und es nangelt der unanfechtbare Beweıs, dass sie auch gegen das naturhliche Vilzbraudcontagıum sielieren Schutz liefert.

Die öffenthıche Gesundheitspflege liegt aber gegen diese Methode noch einıge Befürchtungen. Diejenige, dass sıch dıe Schutzkraft nur auf eınen gewissen Zeıtraum erstreckt, ist von untergeordneter Bedeutung, denn, gelingt es nur einnıal, die übrigen Schwierigkeiten zu beseitıgen, so werden sichı die Schutzimpfungen verhàltnissmässıg so leiclıt und auch wohlfeıl genuz ausführen lassen, um sie in Bedurfsfalle auch jährlıch zu wiederliolen.

Von grösserer Wichtıgkeit ist die Frage, ob nicht das Fleisch, die Milch etc. der geımpften Thiere den Mlzbrand auf den Yenschen ubertragen könne; jedenfalls wirl vor der Verallgemeınerung der Schutzımpfungen auch die Frage zu lösen seln, nach Ablauf welcher Zeit geımpfte Thıere zum Consum zugelassen werden dürfen.

Wenn ıch bedenke, dass der Inpfstoff Milzbrandpilze, wenn auch In mitigirtem Zustande, aber in colussilen Vencen enthält, dass die Pilze im Organısmus der geımpften There eine weıtere riesenlıafte Vermehrung eingelıen, so ergiebt sıch, dass durch die allgemeın ausgefülırten Schutzimpfungen von diesen Pilzen ganz undenkbare Mlengen im ganzen Lande verbreitet würden. Todesfälle werden zu jeder Zett auch unter den geimpften Thieren vorkommen, wenn auch weniger an Milzbrand, so doch an anderen Krankheiten. Es ist nicht ausgeschlossen, dass die aus den Cadavern frei gewordenen Pilze, wenn sie zerstreut werden, ihre ursprüngliche Virulenz auf irgend eine Weise zurückgewinnen, und dass sıe Thuere, inshesondere aber den Menschen - trotz der Mühen und liosten - auf diesem Umwege inficıren können, was umsomehr zu befürchten steht, als die Sorglosıgkeit, mit welcher man schon jetzt selbst die Mllzbrandcadaver behandelt, dann, im Glauben an die Allmacht der Schutzimpfungen - soweıt möglich - noch zunehmen wirl.

Alles das erwogen, pflichte ich der Meinung der Conımission vollkommen bei, dass die sofortige Verallgeneinerung der Pasteur' schen Methode in der uns hier demonstrirten Form verfrüht, dass es an allerwenigsten zu empfehlen ware, sie unter dem Schutze der Staatsautorität zu verbreiten und dass mıt Rücksicht auf die möghichen anderen santären Schäden - Privaten die Ausfülırung von Sclutzımpfungen auf elgene Faust sogar zu verbieten und nur unter der Bedingung zu gestatten wäre, dass sıe sie von eınem staathchen Sachiverstandıgen und unter seıner Beaufsichtigung vornelımen lassen.

Die Commission ist weit davon entferat die Pasteur sche Nethode endgültıg zu verwerfen. Sie ist der Meinung, dass wohl auch. durch eıne strenge sanitätspolizeilıche Regelung des Verfalırens mıt den an Mhlzbrand erkrankiten Thieren und deren Leıchenthelen, gegen die Vermelırung des hrankheitsstoffes und die Verbrettung der Seuclie sehr viel zu erreichen wäre, dass aber den Schutzımpfungen ihr allgemeiner Werth auclı dann, insbesondere beinn en- und epizootıschen Auftreten des Milzhrandes gewahrt bhebe, und speciell die Pasteur sche Methode betreffend ist sie der Meinung, dass lire schon jetzt Levorstehenden Erfolge zu den besten Hoffuungen auf dı Yoglıchbeıt der wünschenswerthen Vervollkommnung berechtıgen. Deshalb hat die Commıssion dem $\mathrm{MI}_{1}$ nisterium anempfohlen, es pö̈ge bezüghtıch der Bereitung des lmpfstofles, der zwecknässigen Ilodificationen der Yethode und der mit der Sehutzimpfung zusammenhängenden Fragen wettere Versuclıe anstellen lassen, zu deren Ausfülırung sie sich eriötig mache und fur welche sie auch eınen Kostenvoranschliag eingereicilıt liat. 